\section{Erlotinib bei NSCLC: Niedrigere Dosis möglich}

\author{
Erlotinib wird meist in der empfohlenen täglichen Dosis von 150 \\ $\mathrm{mg}$ verabreicht. Dies ist die maximal tolerierte Dosis. Nun wurde \\ die Wirksamkeit niedrigerer Erlotinib-Dosen untersucht.
}

$\mathrm{V}$ iele neue Substanzen wie Erlotinib werden zur Anwendung in ihrer maximal tolerierten Dosis entwickelt. Das von zytotoxischen Chemotherapien abgeleitete Vorgehen ist aber nicht unbedingt auf zielgerichtete Substanzen übertragbar, denn diese können möglicherweise ihre maximale Wirksamkeit bereits bei niedrigeren Dosen erreichen. Für Erlotinib wurde nun untersucht, welche Wirksamkeit eine niedrigere als die maximal tolerierte Dosis von 150 $\mathrm{mg} / \mathrm{d}$ hat. In der Datenbank des DanaFarber Cancer Institutes in Boston, MA/ USA, wurden 198 Patienten mit fortgeschrittenem nichtkleinzelligem Lungenkarzinom (NSCLC) identifiziert, die EGFR-Mutationen hatten und mit Erlotinib behandelt wurden. Der Therapieverlauf einschließlich der Erlotinib-Dosis zu
Therapiebeginn, nach 4 Monaten und bei Krankheitsprogression wurden retrospektiv untersucht. Das progressionsfreie Überleben (PFS) von Patienten unter der maximal tolerierten Dosis (150 $\mathrm{mg} / \mathrm{d}$ ) und solchen unter reduzierten Dosen $(\leq 100 \mathrm{mg} / \mathrm{d})$ wurde verglichen.

$16 \%$ der Patienten begannen die Therapie mit einer reduzierten ErlotinibDosis von $\leq 100 \mathrm{mg} / \mathrm{d}, 84 \%$ erhielten die maximal tolerierte Dosis von $150 \mathrm{mg} / \mathrm{d}$. Patienten mit reduzierten Dosen waren im Durchschnitt älter (71 vs. 60 Jahre; $p=0,001)$ und hatten einen niedrigeren Perfomancestatus $(p=0,01)$ als Patienten, die zu Beginn 150 mg/d bekamen.

$77 \%$ der Patienten sprachen auf reduzierte Dosen an. Damit war die Gesamtansprechrate mit den bisher beobachteten Ansprechraten unter 150 mg/d
Erlotinib vergleichbar. Das mediane PFS von Patienten mit reduzierten ErlotinibDosen bei Therapiebeginn betrug 9,6 Monate versus 11,4 Monate bei Patienten, die $150 \mathrm{mg} / \mathrm{d}$ erhielten. Der Unterschied war nicht signifikant (Hazard Ratio 0,81; $p=0,30$ ). Bei Patienten mit reduzierten Erlotinib-Dosen bestand ein nicht signifikanter Trend zu höheren Progressionsraten im ZNS.

Fazit: Auch bei Dosen unterhalb der maximal tolerierten Dosis führte die Therapie mit Erlotinib zu einer hohen Ansprechrate und einem verlängerten medianen PFS. Eine Literaturübersicht zeigt, dass für 15 von 30 zugelassenen oder in der Entwicklung befindlichen niedermolekularen Inhibitoren (,small molecule inhibitors") Dosierungen unterhalb der maximal tolerierten Dosis empfohlen werden.

Judith Neumaier

Lampson BL et al. Activity of Erlotinib When Dosed Below the Maximum Tolerated Dose for EGFR-Mutant Lung Cancer: Implications for Targeted Therapy Development. Cancer 2016;122(22):3456-63.

\section{NSCLC: Weiter Weg zur personalisierten Medizin}

In der BATTLE-2-Studie wurde die Wirksamkeit verschiedener zielgerichteter Therapien untersucht.

$\mathrm{M}$ it der BATTLE-Studie (Biomarkerintegrated Approaches of Targeted Therapy for Lung cancer Elimination) gelang beim nichtkleinzelligen Lungenkarzinom (NSCLC) ein großer Schritt in Richtung personalisierte Medizin. Dort wurde den Patienten eine Biopsie entnommen und diese auf bestimmte Biomarker untersucht, anschließend erfolgte eine adaptive, auf Biomarkern basierende Randomisierung.

Darauf aufbauend wurde nun die Umbrella-Studie BATTLE durchgeführt, um die Wirksamkeit zielgerichteter Therapien mit Fokus auf KRAS-mutierte Tumoren zu untersuchen. Patienten mit fortgeschrittenem NSCLC (und keinen aktivierenden EGFR-Mutationen oder ALK-Genfusionen), die refraktär auf mehr als 1 Vortherapie waren, wurden randomisiert und stratifiziert nach ihrem KRAS-Status in 1 von 4 Gruppen eingeteilt. Genexpressionsprofile wurden durch Targeted-Next-GenerationSequencing erstellt, mit dem Ziel, prädiktive und prognostische Biomarker zu untersuchen. 200 Patienten $(27 \%$ mit KRAS-mutierten Tumoren) erhielten nach adaptiver Randomisierung entweder Erlotinib (Arm A: $n=22$ ), Erlotinib plus MK-2206 (Arm B: $n=42$ ), MK-2206 plus AZD6244 (Arm C: $\mathrm{n}=75$ ) oder Sorafenib (Arm D: $n=61$ ). Primärer Endpunkt war die Krankheitskontrollrate nach 8 Wochen. Diese betrug in der gesamten Population $48 \%$, unter Erlotinib allein $32 \%$, in Arm B $50 \%$, in Arm C $53 \%$ und unter Sorafenib $46 \%$. Bei Patienten mit KRAS-Mutationen lagen die Raten bei $20 \%, 25 \%, 62 \%$ und $44 \%$, bei KRAS-Wildtyp bei $36 \%, 57 \%, 49 \%$ und $47 \%$.

Das mediane progressionsfreie Überleben betrug 2,0 Monate, unabhängig vom KRAS-Status (Arm A: 1,8 Monate, Arm B: 2,5 Monate, Arm C: 2,2 Monate, Arm D: 2,1 Monate; $p=0,17$ ). Das mediane Gesamtüberleben betrug bei Patienten mit KRAS-Wildtyp 6,5 Monate (9,0 in Arm A und B vs. 5,1 Monate in Arm $C$ und $D ; p=0,03$ ). Bei Patienten mit mesenchymalen Tumoren betrug das mediane Gesamtüberleben 7,5 Monate im Vergleich zu 5 Monaten bei epithelialen Tumoren $(p=0,02)$. Die Toxizitäten, v. a. der neuen Therapien, entsprachen denen in früheren Untersuchungen.

Fazit: Trotz eines verbesserten progressionsfreien Überlebens unter einer Therapie ohne Erlotinib bei Patienten mit KRAS-Mutationen und einer verbesserten Prognose bei mesenchymalen Tumoren besteht weiterhin Bedarf an besseren Therapiestrategien, die Biomarker berücksichtigen.

Judith Neumaier

Papadimitrakopoulou V et al. The BATTLE-2 Study: A Biomarker-Integrated Targeted Therapy Study in Previously Treated Patients With Advanced Non-Small-Cell Lung Cancer. J Clin Oncol. 2016;34(30):3638-47. 